\title{
Fundamental basis, informational meaning and data analysis of multifrequency reoencephalography
}

\author{
Yu. Moskalenko ${ }^{1,}$, G. Weinstein ${ }^{1}$, T. Kravchenko ${ }^{3}$, N. Ryabchikova ${ }^{2}$, Yu. Andreeva ${ }^{1}$, \\ V. Semernia ${ }^{1}$ \\ ${ }^{1}$ Institute of evolutionary physiology and biochemistry Russian Academy of Sciences, St.Petersburg \\ ${ }^{2}$ Moscow State University, Moscow \\ ${ }^{3}$ Russian School of Osteopathic Medicine, St.Petersburg, Russia
}

\section{Email address :}

yurimos@mail.ru(Yu. Moskalenko)

\section{To cite this article:}

Yu. Moskalenko, G. Weinstein, Yu. Andreeva, N. Ryabchikova, V. Semernia, T. Kravchenko. Fundamental Basis, Informational Meaning and Data Analysis of Multifrequency Reoencephalography. Advances in Bioscience and Bioengineering. Vol. 1, No. 1, 2013, pp. 8-21.

doi: 10.11648/j.abb.20130101.12

\begin{abstract}
A long historical experience of monofrequency rheoencephalography (REG) showed its limited capabilities. The new approach is presented - multifrequency REG that is REG recording simultaneously at three frequencies - 16, 100 and $200 \mathrm{kHz}$. The different brain impedances received were analyzed on the base of equivalent electrical circuits of brain tissue. First, this enables to calculate intra-extracellular electrical component for evaluation of brain tissue hydration state by means of an original computational method. Second, dynamic cooperative analysis of multi-REG and transcranial Doppler pulsations provides the indices of intracranial CSF mobility (CSFm) and cranial compliance (CCe). Third, spectral analysis of processes recorded provides the new valuable information about regulatory processes and brain metabolism involved, and this enables to evaluate indirectly brain cognitive capabilities. Fourth, comparison of spectrum diagrams of slow intracranial volume fluctuations in ranges $0-0.3 \mathrm{~Hz}$ demonstrates their similarity both in healthy human and in awake rabbits, notwithstanding their rates differences of heart pulsations and respiratory chest movements. The application of this new approach to investigation of intracranial circulation in healthy humans of different ages and in neurosurgical patients showed its promising efficacy.
\end{abstract}

Keywords: Rheoencephalography, Multifrequency Brain Impedance, Brain Hydration, Slow Intracranial Fluctuations in Human and Rabbits, Spectral Analysis

\section{Introduction}

In the middle of the Twentieth Century two alternative methods were developed for the study of cerebral circulation. One of them was based on the clearance effect which allowed progressive measurements of cerebral blood flow in absolute units ( $\mathrm{ml}$ of blood flow per 100gr of brain in $1 \mathrm{~min}$ ) within intervals of a few minutes [1]. The second was derived from a method based on measurement of electrical impedance of biological tissues or more specifically, monitoring of electrical resistance between electrodes placed on different regions of the human body, including the head which had first been known as electroplethysmography and then as rheography [2,3]. Depending on the body region, modifications of this method were named after the specific organ being investigated. In the context of the present paper, dealing specifically with investigations of fluid circulation through the brain, the term Rheoencephalography or REG will be used. The advantage of this method is that it is high dynamic. The obvious disadvantage is the data received can only be evaluated comparatively.

\subsection{Fundamental Background of Monofrequency REG}

The development of this method has a complicated history. In the middle of the Twentieth Century this method was applied to the study of cerebral circulation in both humans and animals $[4,5,6,7]$. Early application of REG methods began in 1958, when the first fundamental studies appeared $[8,9]$. In these investigations the foundations of the method were established, based on the fact that specific electrical conductivities of the main intracranial components - brain tissue, blood and cerebrospinal fluid 
(CSF) - correlate with the following ratio --1:0.5:0.1, which has been corroborated in recent investigations [10]. Just after the fundamental studies were completed, mathematical simulation verified that the changes of electrical resistance between electrodes placed on the head are proportional to blood/CSF volume relationships in the region between electrodes. These calculations also showed that changes of electrical resistance between electrodes, which depend on blood-CSF volume redistribution, are rather small. Their ranges are $1-1.4 \%$ of the total value of the electrical impedance between the electrodes. The changes depend on the frequency of the electrical current being applied to the transmitting electrodes and some other factors. The complex biophysical analysis of the role of all factors forming the pulse wave and similar fluctuations of electrical resistance between electrodes - specific resistance of the brain tissue, blood, and CSF, as well as the processes of polarization at the contact point between the metal electrode and skin connection was summarized in the book "Dynamics of Brain Blood Volume Under Normal Conditions and Gravitational Stresses" [11]. These studies established that the optimal frequencies for REG recording of the human brain range between 80 and $100 \mathrm{kHz}$. Later these frequencies became the accepted norm by the majority of REG instrument manufacturers through the world.

Since the middle of the1960's REG began to be used in clinical investigations for diagnosis of cerebrovascular pathology and shortly thereafter some guidelines appeared suggesting values for the norm and what significance any deviation might indicate.[12,6]. Because objective analysis of changes of REG pulsations appeared to be a quite complicated task, numerous (about 50) different empiric criteria for REG pulse wave deformations were developed.

However, only a few of them were proven to have an important physiological background. Maximal amplitude, slope of anacrotic increase, and anacrotic-dicrotic phase ratio, for example, were substantiated and only these were retained for use in common clinical practice. After fifteen years of clinical experience using REG methods around the world the consensus opinion for its future was not optimistic. The method had proven to be indirect, sensitive to numerous interferences depending on head and body movements and additionally the diagnostic significance of REG remained ambiguous. Consequently by the end of the 1970's the usefulness of this method had to be reevaluated. A careful analysis of REG methodology was performed [13]. This review of the methodology and literature also didn't present an optimistic picture for the future of REG. The review established that it is impossible to measure CBF indices or to provide a medical diagnosis by REG alone, because numerous peculiarities of the REG pulse wave are not directly connected with $\mathrm{CBF}$ but to a great extent are influences from external factors. Therefore any conclusions based on the wave form of the REG recordings must be questionable. Nevertheless, some changes of REG pulse pattern deformation, for example, the appearance of small additional waves in the dicrotic phase were recognized as characteristic for arterial hypertonic disease. On the brighter side it was definitely established that REG, when combined with functional tests (apnoe, hyperventilation, Valsalva and Stookey tests), could provide some indices of cerebrovascular reactivity (CVR). One of the shining successes of REG application was when studying CVR changes in astronaut crews during different stages of space missions as compared to pre-flight investigations [14]. These investigations were accepted as fundamental principles for the reaction of the cerebrovascular system to space flight conditions [15]. During the ensuing years not a single new significant bit of data concerning the informational meaning of REG appeared with the exception that some animal investigations showed REG could be used for drug screening and for evaluation of the limits of CBF autoregulation [16]. General interest in this method decreased.

\subsection{Ways to Increase of REG Informational Meaning}

Historical experience with application of REG indicates that this method as a stand-alone instrument provides very limited information useful for physiology or for clinical practice because the wave form reflects volume distribution of both blood and CSF and the signal also contains some

significant interferences originating from the subject's eye, throat, lip and face movements and larger movements of their body. This suggests that broadening the informational meaning of REG should be based on coupling it with another instrument which also reflects the_dynamic component of intracranial fluid circulation but which is based on some other physical principle. In the 1980's the possibility of such an instrument first appeared with the advent of transcranial Dopplerography (TCD) [17]. TCD promised to be the most acceptable partner for the REG methodology.

\subsection{Coupling REG and TCD Methods}

By combining the REG and TCD method it is possible to measure pulsations of blood flow velocity simultaneously with the REG wave pulsations. Both methods are capable of monitoring changes during very small increments ofthe cardiac cycle and their wave forms are synchronous. Investigations carried out using the combination of REG and TCD along with computer processing of their dynamic relation have shown this methodical combination can determine, in comparative units, both CSFmobility in the craniospinal cavity and pulse changes of intracranial volume which represents the dynamic component of Cranial Compliance (CCe) [18, 21]. More specifically, this presents the possibility to monitor comparative changes of the distribution of fluids between extracellular spaces and brain tissue under different conditions. During the last years when this partnering to TCD was reinvigorating the stature of REG as an instrument to monitor brain fluid dynamics, advancements in electronic engineering gave the basic REG instrument a revolutionary new possibility. This 
development also has an interesting historical background.

About 50-ty Years ago simultaneous observations of the comparative changes of the capacitance and resistance components of electrical impedance of applied primary to the human mammary gland during lactation and demonstrated the possibilities to reveal gland dysfunction during the first postnatal days due to milk congestion. Steady state impedance values could reveal peculiarities of milk distribution between extracellular spaces and inside the cells of the secretory glands and thus suggest the appropriate remedy-either hot or cold compresses [22]. These investigations have shown the way to apply this principle to the realm of brain fluid monitoring suggested that the capacitance component of brain tissue impedance may be of value for determination of the brain hydration level. The background of this statement is based on animal investigations with electrodes implanted into the skull which revealed the relation between the capacitance component of brain electrical impedance and the state of brain tissue hydration [23, 24]. Recently, during animal investigations using a hypoxic model it was shown that relations of capacitance and resistance at different frequencies develop differently during hypoxic reaction due to brain tissue hydration $[10,25]$.

\subsection{Multifrequency REG: Background and Advances}

All these investigations stimulated the next stage of REG development - recording of REG, using not one but three or four different frequencies simultaneous from comparatively low to high electrical current which could be realized only recently on the base of modern microelectronics and computer processing. The advantage of this REG modification - now called multi-REG - consists in that, depending on the frequency of current used for head impedance measurement, different components of intracranial media are involved in the electrical current conductivity. Firstly, this gives the possibility on the base of values of basic impedance, measured simultaneously on different frequencies, to calculate components of the simplest equivalent electrical circuits of brain tissue first presented many decades ago [26]. This equivalent circuit is composed of a combination of three elements - two resistive and one capacitance. Measured values of basic resistance on three frequencies between the head electrodes allows for the calculation of its components and thus non-invasive evaluation of intracranial passive electrical properties under different physiological and pathophysiological conditions.

Secondly, multi-REG in combination with TCD allows for calculations of CSF mobility components by the method described earlier [18] and to calculate slow fluctuations of intracranial components $(0.1-0.3 \mathrm{~Hz})$, which reflect activities of cerebrovascular autoregulatory mechanisms [27]. Additionally the informational meaning of the slow fluctuations could be increased by comparison of their amplitudes and rate, at different REG frequencies.

Therefore, the present paper has multiple aims: 1) to describe the biophysical background, and informational meaning of the multi-REG as a stand-alone; 2) to describe the additional informational possibilities due to simultaneous recording with $\mathrm{TCD}$; 3) to describe the principles of automating the analysis of the data received; 4) to demonstrate the informational meaning and perspectives of spectral analysis of slow 5 - 15 cycles/min fluctuations of intracranial volume, reflected by multi-REG in both human observation and animal experiments.

\section{Methods}

Description of the methodical aspects of the multi-REG includes the principles of selection of frequencies, methods for evaluating the values received from each single component of the equivalent circuits between the electrodes placed on the human head and presentation of the results received, including the principles of automated analysis of each fragment.

\subsection{Optimal Physical Conditions of Multi-REG Recording}

The selection of the optimal frequencies for the multi-REG design is based on studies of the dependence of impedance frequencies on different kind of tissues. It has been established that changes of dielectric resistance of tissues is not critically dependent on frequency in the range from 50 to $500 \mathrm{kHz}$. For instance, for intracranial liquids it is $0 \%$ for CSF and up to $9 \%$ for blood, for brain tissues 8 $-9 \%$; and for skull bones about $10 \%$ [10] but the complex impedance for the whole head varies nearly twice as much over the above frequency ranges[11, 24]. This means that the impedance values between electrodes placed on the human head at each frequency are determined by the distribution of the electrical current around or through the particular tissues of the head. From this point of view the frequency ranges for the multi-REG should be as wide as possible. However, the low frequencies of electrical current the limitation is determined by the impedance of extracranial tissues (skin, muscle, fat, membranes ), as well as the electrical sensitivity of living tissues, and so a reasonable low frequency limit would be not less than 15-20 $\mathrm{kHz}[11]$.

Suggestions about the upper frequency limits have been presented in the numerous publications, which indicate these limits are determined by several factors. The frequency ranges should be selected from within the frequency band where there is a linear dependence on intracranial tissue impedance and minimal influence of external and perhaps internal electromagnetic fields, and of parasitic capacitances. Based on these suggestions the optimal level of the upper frequency limit for the multi-REG should be about $200 \mathrm{kHz}$.

Thus, the most acceptable range for the multi-REG should be from 15 to $200 \mathrm{kHz}$. The lower and upper frequencies should be selected at these points. Taking into account the peculiarities of general frequency dependence 
of intracranial media, the third frequency should be in the vicinity of $100 \mathrm{kHz}$. These considerations have been taken into account during design and construction of a prototype version of the multi-REG instrument manufactured by "Mitsar-Medical", St. Petersburg, Russia.

\subsection{Instrumentation}

The instrumental complex used in our evaluation of the possibilities and limitations of a multi-REG unit as a basic device for physiological and pathophysiological investigations as well as for clinical examinations was this new version of the multi-REG instrument mentioned above. For data collection, the analogous- digital transformer "PowerLab-5" from ADInstrument was used with all its software functions including low frequency spectrum analysis. Multi-REG signals were received by three silver plate-electrodes, each one $20 \mathrm{~mm}$ in diameter, placed on the human head. A common electrode was placed on the frontal region and two were placed mastoidaly behind the left and right ears. Construction of the multi-REG instrument allows recording all three frequencies signals using just one wire for each electrode. Other equipment used was a two channel transcranial Doppler (DWL Germany) to record linear blood flow velocity at the base of the middle cerebral artery and respiratory transducer for recording chest respiratory movements (ADI-MLT 1132). All devices were controlled by PCs running "Windows-XP". Methods of recording and analyzing the Doppler/REG signals to evaluate CSF mobility have been described earlier [18]. In all investigations signals from the above-mentioned instruments were received onto a multi-channel recording board. Two channels were for right and left hemisphere TCD, six channels were for right and left hemisphere multi-REG pulsations channels (fluid pulsations using three frequencies from each hemisphere) and 3 channels from each hemisphere for DC signals indicating basic impedance values and their possible changes during the investigations. An additional channel was used for recording respiratory chest movements, which is important to differentiate spectrum values of intracranial CSF fluctuations from respiratory chest movements because they sometimes occur at similar frequencies. After the investigations were concluded the most interesting fragments of the recordings were analyzed. It is important to emphasize, that for spectrum analysis "PowerLal-5" software used, which is founded on "fast Fourier transformer". For analysis it is necessary to have at least a continuous two minute segment of recording without interferences due to the subject's facial muscle movements, etc. $64 \mathrm{k}$ quantitation have been used for spectral diagram representation in frequency interval $0-$ $0.3 \mathrm{~Hz}$ and $0-2.8 \mathrm{~Hz}$.

\subsection{Providing of Investigations}

The investigations were carried out on three aging groups of healthy persons $(16-24$ years $(n=31), 25-50$ years $(n=39)$ $51-90$ years $(n=49)$, and 8 neurosurgical patients investigated before surgery and during 10 postoperative days The data received were analyzed in three ways: Calculations of resistance and capacitance components of the equivalent circuits shown below (Fig.1), on the base of measured impedance levels between electrodes at different frequencies was provided.

Significant advance of REG methodology may be based on coupling multiREG with REG-TCD recordings. However for this it is necessary to automate CCe - CSFm calculation, because manual method, described above [18], is not simple in realization. Automation helps calculate the indices representing CSF mobility (CSFm), dynamic cranial compliance (CCe). At this type of analysis the pulse fluctuations of REG and TCD recorded at100 kHz were used.

Spectrum diagrams from artifact-free fragments lasting 90-120s from all three multi-REG frequencies were presented at $0-0.3 \mathrm{~Hz}$ together with TCD pulse spectrum analysis and respiratory chest movements for excluding slow the fluctuations not of intracranial origin; thus fluctuations in TCD spectrum specific to the cardiovascular system and those fluctuations driven by the respiratory system could be identified in the multi-REG spectrums.

With the purpose to compare the indices of intracranial slow fluctuations in humans and in other mammalian representatives, 6 experiments were also provided with awaked rabbits. Plate $0.2 \mathrm{~cm} 2$ electrodes were placed bi-temporally on depilated animal head skin resembling the human REG positions, and the analogous REG fragments were recorded and analyzed.

\subsection{Analysis of Equivalent Electrical Circuits}

Measurements of basic resistance between electrodes are possible to calculate by parameters of the most simple head tissue equivalent circuit diagram. Indeed, from the electrical point of view, brain tissue may be represented as a set of resistances and capacities intricately connected to each other. However, the equivalent circuit of the brain is simple (fig. 1). The frequency dependence of brain impedance is:

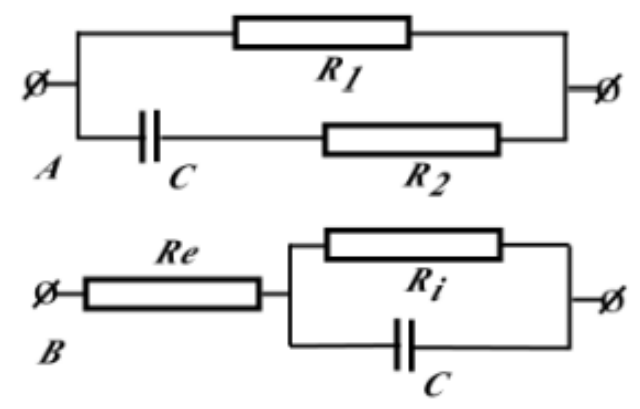

Figure 1A. Equivalent circuit of the brain (Schwan, 1957) (Circuit I). R1 suggests the extracellular resistance, $R 2$ - intracellular resistance, and $C$ the cellular electrical capacitance.

Figure 1B. Equivalent circuit of the brain (Circuit 2). Re is the extracranial resistance, $R i$ - intracranial resistance, and $C$-cellular electric resistance. 


$$
Z=\frac{R_{1}\left(R_{2}+\frac{1}{i \omega C}\right)}{R_{1}+R_{2}+\frac{1}{i \omega C}},
$$

where $\mathrm{Z}$ is the brain tissue impedance; $\mathrm{R} 1$ is the extracellular resistance; $\mathrm{R} 2$ is the intracellular resistance and $\mathrm{C}$ is the cellular electric capacitance; $\omega$ is the angular frequency; $\mathrm{i}$ is the imaginary unit $i=\sqrt{-1}$. The equivalent electrical circuit of the brain represented in fig.1(circuit 1) is not exclusive. Brain tissue may be described otherwise (fig 1b). In this case brain impedance is calculated as

$$
Z=\frac{\left(R_{e}+R_{i}\right)+i \omega C R_{e} R_{i}}{1+i \omega C R_{i}},
$$

where $\mathrm{Z}$ is the brain tissue impedance; $\mathrm{Re}$ is the extracranial resistance, $\mathrm{Ri}$ is the intracranial resistance and $\mathrm{C}$ is the cellular electric capacitance; $\omega$ is the angular frequency.

However, the above brain circuits don't allow us to determine the contribution of extracranial resistance and extracellular brain resistance simultaneously. Future progress in this mode of calculation, which could permit such data to be receive, could be based on including one more frequency, for example $50 \mathrm{kHz}$, to the number of frequencies now being measured. Currently estimation may be performed by interpolation methods. By having three measurements at three various frequencies we can approximate the impedance function with a simpler function Z(f). Hereafter we can estimate the impedance value at the fourth frequency with function $Z(f)$. The calculations as presented substantiate the conclusion that the transcephalic measurement of electrical impedance that has been used in these investigations is a measurement of the electrical impedance of the whole head as the volume conductor from the head surface by a noninvasive method.

Realization of multi-REG investigations was performed by computations of electric impedance parameters, using the special program 2007solve.xls written in Visual Basic for Applications language (VBA). This program is integrated in Microsoft Excel 2007 workbook. The program allows us to compute automatically, values of impedance parameters (R1, R2, C in circuit-Fig 1a and Re, Ri, C in circuit Fig.1b). Input data for 2007solve.xls are values of impedance measured at three distinct frequencies $(16 \mathrm{kHz}$, $100 \mathrm{kHz}, 200 \mathrm{kHz}$ ). The least squares method can be used to estimate the parameters of impedance magnitude. The problem of finding the impedance parameters is reduced to finding the minimum of regression sum:

$$
S=\sum_{i=1}^{3}\left(|Z|_{i}-I_{i}\right)^{2}
$$

minimum of the regression sum we use the quasi-Newton BGFS method, which is programmed into our 2007solve.xls file. These manipulations allow us to evaluate different brain impedance parameters in each of the circuits

Coupling of multi-REG or monofrequency $(100 \mathrm{kHz})$ REG with TCD could significantly expands the field of REG application and increase its informational meaning, because this gives the possibility to obtain information about CSF-mobility (CSFm) and skull biomechanics properties (CCe).Manual calculations of these indexes [18] were shown to provide an exact and valuable information, but this method consumes too much labour, time and attention. Automation significantly facilitates this process and could give the acceptable results, as it was described separately $[19,20]$.

Thus, combination of multi-REG calculation of electrical resistance distribution in human head with coupling REG $(100 \mathrm{kHz})$ and TCD, permits significantly expand the ways for application of REG to applied physiology, neurology and neurosurgery as an valuable noninvasive, comparatively simple and dynamic method for investigation of cerebral blood flow and CSF systems and of skull biomechanics.

\section{Results}

Examination of the informational meaning of new multi-REG method was performed in two ways. One was carried out on healthy volunteers of three aging groups during rest conditions. The second approach was the observations of neurosurgical patients with symptoms of brain edema during the first 5 - 7 days after the surgery. Additionally, the experiments on awake animals were carried out using the same, as in humans, methodical complex and similar methods of spectrum analysis.

\subsection{Estimation of Basic Values of Parameters of Equivalent Circuits}

The results of age related measurements of human head impedance at different frequencies are summarized in Table.1. The results of calculations are summarized in Table.2. For detailed analysis of the resistance and capacitance components of equivalent circuits 1 and 2 their values were grouped for different ages and coupled with the CCe-CSFm plot diagram, constructed with the data obtained and calculated at $100 \mathrm{kHz}$ and represented in Fig.2. The frequency of $100 \mathrm{kHz}$ was selected for calculations of CCe-CSFm plot because all our previous calculations were provided at this frequency. The recent research has confirmed this selection, because comparative values of $\mathrm{CCe}$ and CSFm at $16 \mathrm{kHz}$ and $200 \mathrm{kHz}$ are about $5-20 \%$ lower than similar data at $100 \mathrm{kHz}$. These data once again confirm that the selection of $100 \mathrm{kHz}$ is optimal for REG observations.

The data presented in Fig.2 shows that there is a significant difference between the components of the equivalent circuits for each age group. This demonstrates 
that such a method of data presentation is significantly more sensitive as compared to calculations of head impedance measurements based on a "perturbative index" [28, 29].

These investigators suggest that the numeric values of cerebral electrical impedance were symmetric in the two brain hemispheres at different frequencies $(20 \mathrm{kHz}, 50 \mathrm{kHz}$ and $100 \mathrm{kHz}$ ) and that age, sex and the time of monitoring have no obvious effect on the results of measurements. However, in our measurements presented above, basic impedance and its pulsing components are different for each investigated age group and, as was shown in earlier investigations, human head impedance is characterized by hemispheric asymmetry [30]. Common to our data and the data referenced above is that the values of impedance between electrodes at rest conditions were comparatively stable during the investigations. Additionally our investigations determined the value of impedance between electrodes changed much less than $1 \%$ during two hours of observation.

Table 1. Age and frequency dependent of averaged impedances:

\begin{tabular}{|c|c|c|c|c|c|c|}
\hline \multirow{2}{*}{ Age groups Age groups } & \multicolumn{3}{|c|}{ Left hemisphere } & \multicolumn{3}{|c|}{ Right hemisphere } \\
\hline & $\mathrm{Z16}, \mathrm{Ohm}$ & Z100, Ohm & $\mathrm{Z} 200 \mathrm{Ohm}$ & $\mathrm{Z16}, \mathrm{Ohm}$ & Z100, Ohm & $\mathrm{Z} 200, \mathrm{Ohm}$ \\
\hline $16-25$ yeas & $406.7 \pm 16.0$ & $301.6 \pm 10.9$ & $286.0 \pm 10.1$ & $398.4 \pm 14.0$ & $298.3 \pm 10.8$ & $282.9 \pm 10.5$ \\
\hline $25-50$ yeas & $406.9 \pm 9.7$ & $312.0 \pm 6.3$ & $298.3 \pm 6.2$ & $420.7 \pm 12.0$ & $318.2 \pm 8.7$ & $305.7 \pm 8.15$ \\
\hline $50-90$ yeas & $424.7 \pm 10.8$ & $320.2 \pm 7.7$ & $307.0 \pm 8.7$ & $418.2 \pm 9.5$ & $324.0 \pm 7.9$ & $303.4 \pm 9.5$ \\
\hline
\end{tabular}

The impedance values were measured at 16, 100 and $200 \mathrm{kHz}$ (mean values \pm St Err.). Z16=impedance at $16 \mathrm{kHz}, \mathrm{Z} 100=$ impedance at $100 \mathrm{~Hz}$, $\mathrm{Z} 200=$ impedance at $200 \mathrm{kHz}$ ) in different age groups

Table 2.R1, R2 an d C values calculated on the base of equivalent electrical brain circuits I and II

\begin{tabular}{|c|c|c|c|c|c|c|}
\hline \multirow{2}{*}{ Age groups Age groups } & \multicolumn{3}{|c|}{ Circuit I. } & \multicolumn{3}{|c|}{ Circuit II . } \\
\hline & R1, Ohm & $\mathrm{R} 2, \mathrm{Ohm}$ & $\mathrm{C},{ }^{*} 10 \mathrm{pF}$ & $\mathrm{R} 1$, Ohm & $\mathrm{R} 2, \mathrm{Ohm}$ & $\mathrm{C}, * 10 \mathrm{pF}$ \\
\hline $16-25$ yeas & $428 \pm 16$ & $815 \pm 34$ & $0.405 \pm 0.07$ & $276 \pm 8$ & $705 \pm 24$ & $0.670 \pm 0.06$ \\
\hline $25-50$ yeas & $341 \pm 12$ & $737 \pm 29$ & $0.491 \pm 0.08$ & $343 \pm 5$ & $747 \pm 15$ & $0.550 \pm 0.05$ \\
\hline $50-90$ yeas & $440 \pm 11$ & $866 \pm 66$ & $0.289 \pm 0.07$ & $290 \pm 12$ & $760 \pm 43$ & $0.515 \pm 0.03$ \\
\hline
\end{tabular}

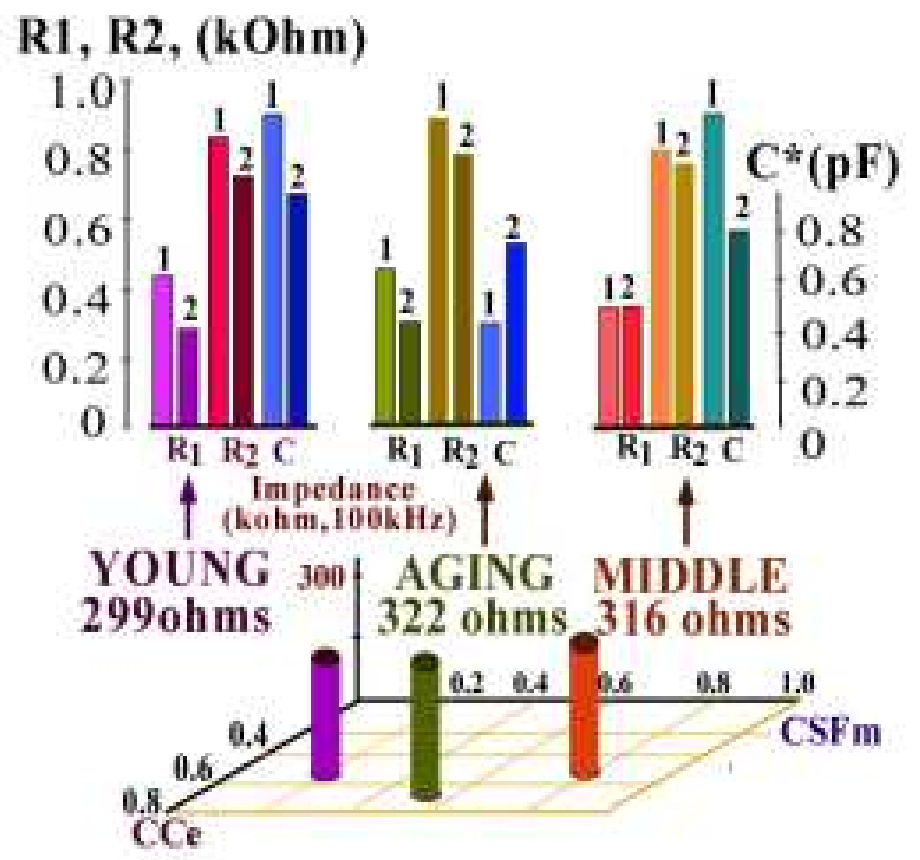

Figure 2. Differences of position on the "field" of the CCe-CSFm plot of the average values of electrical impedance of different Aging groups (measured at $100 \mathrm{kHz}$ ) and the averaged values of the separate of elements of electrical equivalent circuits 1 and 2.

An age dependent values of R1, R2 and C, calculated on the base of equivalent electrical brain circuits I and 2 (see Fig. 1). Values of total electrical impedances were taken

\section{from Table 1.}

Generally, values were of a similar pattern for R1 and R2, but were significantly different for $\mathrm{C}$, as can be seen for the 
aging groups. This suggests that both circuits, in principle, reflect the same peculiarities of resistance distributions between electrodes. Changes of capacitance for the aging groups appear to be connected with structural changes of brain tissue during aging as described in numerous publications [30, 31].

It is important to note the similarity of R1 values for both circuits in all aging groups. This shows, that the influence of surface (circuit 1) and transfer (circuit 2) resistances of extracranial tissues is not as high as had previously been suggested. From the beginning of REG development the supposed influence of comparatively high resistances of extracranial tissues and skull bones had been the primary justification for skepticism about the REG method [33, 34]. In reality, R1 also includes resistances of intracranial factors such as the shunting role of subarachnoid CSF and the brain membranes, the dura mater and others. The values of R2 were similar in all measurements and were about double R1. This means that the brain tissue extracellular spaces play a part in forming the general resistance between electrodes in both circuits, but in circuit I this influence is a little more. Comparatively similar values of $\mathrm{R} 2$ resistance measured in both circuits and in all aging groups shows that extracellular structure of the brain tissue in all aging groups is similar. However, for circuit I the R2 value was a little higher than for circuit II in all aging groups. It suggests that the shunting effect of R1 in circuit I more accurately reflects extracellular resistance. A similar effect is also exerted by the contribution of brain cellular membranes in young and in middle aging groups, but it is significantly different in the elderly group, as can be seen by comparison of capacitance values in all aging groups.

\subsection{Coupling Multi-REG with MonoREG - TCD Investigations}

The latter determined the averaged position of calculated values of $\mathrm{R} 1, \mathrm{R} 2$, and $\mathrm{C}$ in the CCe - CSFm plate for different aging groups. For the young group the values of CCe and CSFm, calculated from multi-REG recordings at $100 \mathrm{kHz}$, were found as: $\mathrm{CCe}=0.80 \pm 0.06, \mathrm{CSFm}=0.30 \pm$ 0.06 ; for middle age group $\mathrm{CCe}=0.49 \pm 0.06$, $\mathrm{CSFm}=0.70 \pm 0.10$; and for elderly group $\mathrm{CCe}=0.83 \pm 0.04$ and $\mathrm{CSFm}=0.47 \pm 0.08$. This means that cranial compliance as well as CSF-mobility for both young and older subjects is comparatively high.

The situation concerned middle-age group is more complicated. In this case some CCe decrease appears to be compensated by CSFm increase. However, the neurological examination shows that in this aging group intracranial compensatory CSF movements are not very active and in some cases are not be fully compensated by CSF mobility. In these cases some neurological dysfunctions might be observed. In the elderly group CCe values increase again and CSFm increases slightly also, owing to special intracranial liquid distribution to assist in brain metabolic supply.
Thus, all the above mentioned data indicate that the informational possibilities of the multi-REG method are greater than the usual s ingle frequency REG. Low levels of St. Err. values shown in table1 and table 2, indicate that under normal physiological conditions intracranial interrelations between liquid media and brain tissue are characterized by comparatively low variations and it explains the comparative stability of data obtained with multi-REG measurements. This is confirmed by the results of the calculations of the elements which compose the equivalent electrical circuits between electrodes placed on the human head. It is necessary to emphasize, that both equivalent circuits proposed are rather primitive, but in general they sufficiently and objectively reflect the special distribution of resistance and capacitance elements. Comparison of the results received by calculations of equivalent circuits I and II give the possibility to reveal a general picture of liquids and brain mass distribution inside the skull.

It is necessary to emphasize, that the equivalent circuits presented above are very simple to compare with the real object of investigation - the human head-because it is already known that equivalent circuit distributions of electrical indices between electrodes may include numerous elements $[10,11]$, but the advantage of the circuits shown in Fig. 1 is the possibility to calculate each single element. Of course, if even one more frequency were added to the multi-REG instrument, it would be possible to increase the number of elements in the equivalent circuits up to four, but due to comparative low increment of frequency dependence of intracranial tissues in the selected frequency ranges, it would not yield significant results. Nonetheless, the data received leads us to conclude that even these simple circuit diagrams present the general picture of the contribution of extracranial and intracranial components measured by multi-REG data. The results of the basic resistance calculations from the circuit diagrams as seen in Fig. 2 add significant informative value to the results of the REG pulsations recorded simultaneously with TCD and analyzed in their direct (time unified) correspondence. Comparison of pulse recordings by REG at three frequencies received simultaneously has confirmed that the most useful frequency is $100 \mathrm{kHz}$ as was established years ago by fundamental experimental animal investigations of REG methodology. Indeed, results of calculations of $\mathrm{CCe}$ and CSFm using other frequencies such as 16 and $200 \mathrm{kHz}$, show that the data received is dynamically similar to the 100 $\mathrm{kHz}$ recordings under different conditions, including functional tests, but their values and variations are significantly less.

\subsection{Spectral Analysis of Low Frequency Multi-REG}

The spectral analysis is a comparatively new way of data representation in cerebrovascular physiology. That is why significant fragment of this investigation is dedicated to this problem. Informational value of spectral analysis follows 
from the fact, that the most of functional activities of physiological systems are accompanied by fluctuations of electrical and nonelectrical nature. At the beginning, the spectral analysis approach was used for analyzing of bioelectrical signals, for example EEG. However, during at least as the last 50 years up to the present time, there were no reliable methods to apply spectral analysis of fluctuations of nonelectric origin, and only recently, due to modern computer technique, it became to be possible.

Application of spectral analysis is the most important for the study of cerebrovascular system and its relation to CSF system and skull biomechanics, because functioning of these systems are accompanied by slow fluctuations $5-15$ cycles per minute, the changes of frequency and amplitude being special for both humans and animals. Therefore the aim of the present section is to show some peculiarities of spectral diagrams for human and animal to attract attention to this new and perspective way of studying of intracranial physiological processes.

For evaluation of fragments of the most informative spectral diagram, spectral analysis of $90-120$ s fragments of REG original records was made (Fig.3, A-1-human and B-1-rabbit were analyzed in frequency ranges $0-2.8 \mathrm{~Hz}$ and $0-0.3 \mathrm{~Hz})$ :

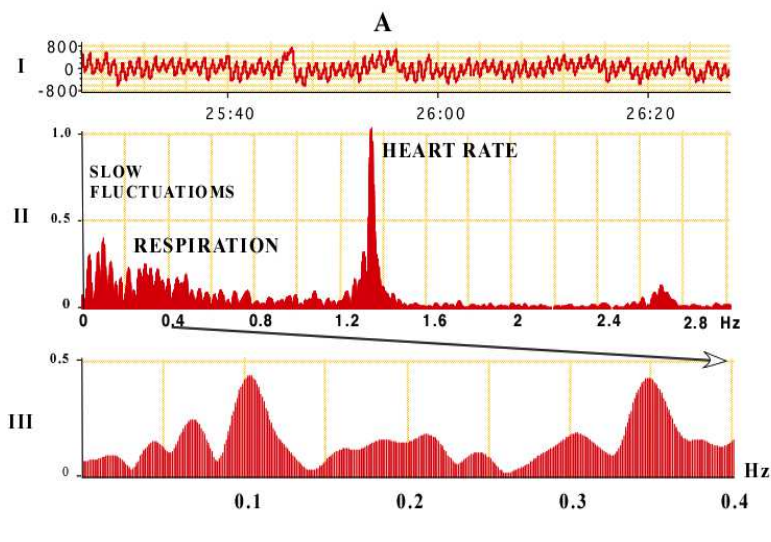

B
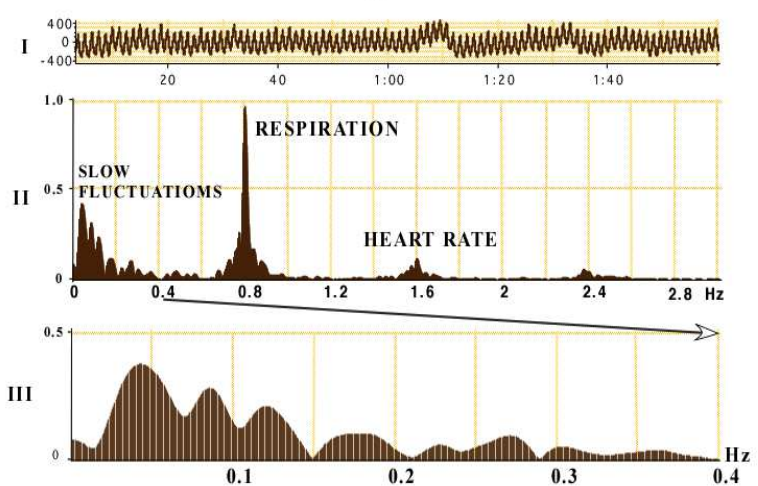

Figure 3. Original REG recordings of human $A-I$ and rabbit $B-I$ and their spectral diagrams in ranges $0-2.8 \mathrm{~Hz}$ (II) and $0-0.3 \mathrm{~Hz}$.

It is of significance, that in spite of different brain size, its physiological organization, heart and respiration rate, the spectral diagrams which reflect slow fluctuations are similar.
Some low frequency peaks at $0,025-0,1 \mathrm{~Hz}$ are similar for human and rabbit. It is reasonable to suggest that both of them reflect regulatory processes of systemic arterial pressure. Fragments of spectrum diagram in ranges $0.15-$ $0.35 \mathrm{~Hz}$ are also similar for human and rabbit and reflect apparently metabolic processes in brain tissue. There is an additional peak $(0.3-0.4 \mathrm{~Hz})$ on human spectral diagram corresponding to respiratory chest movements, but it is absent on animal spectrum.

Detailed analysis of multiREG recordings in frequency ranges $0-0.3 \mathrm{~Hz}$ is shown on Fig. 4 . The differences can be observed in all of the recorded fluctuations of the multi-REG pulses, at 16,100 and $200 \mathrm{kHz}$, in the TCD pulsations and in the fluctuations, which reflect respiratory movements of the chest. They are unique for every frequency as well as for the TCD pulse and they are characterized by a number of peaks, which may be both similar and different for every mentioned fluctuation process. Comparison of peaks from any of the recording devices to peaks on other recording devices can give some indication of their origin. This is possible to see this in Fig. 4

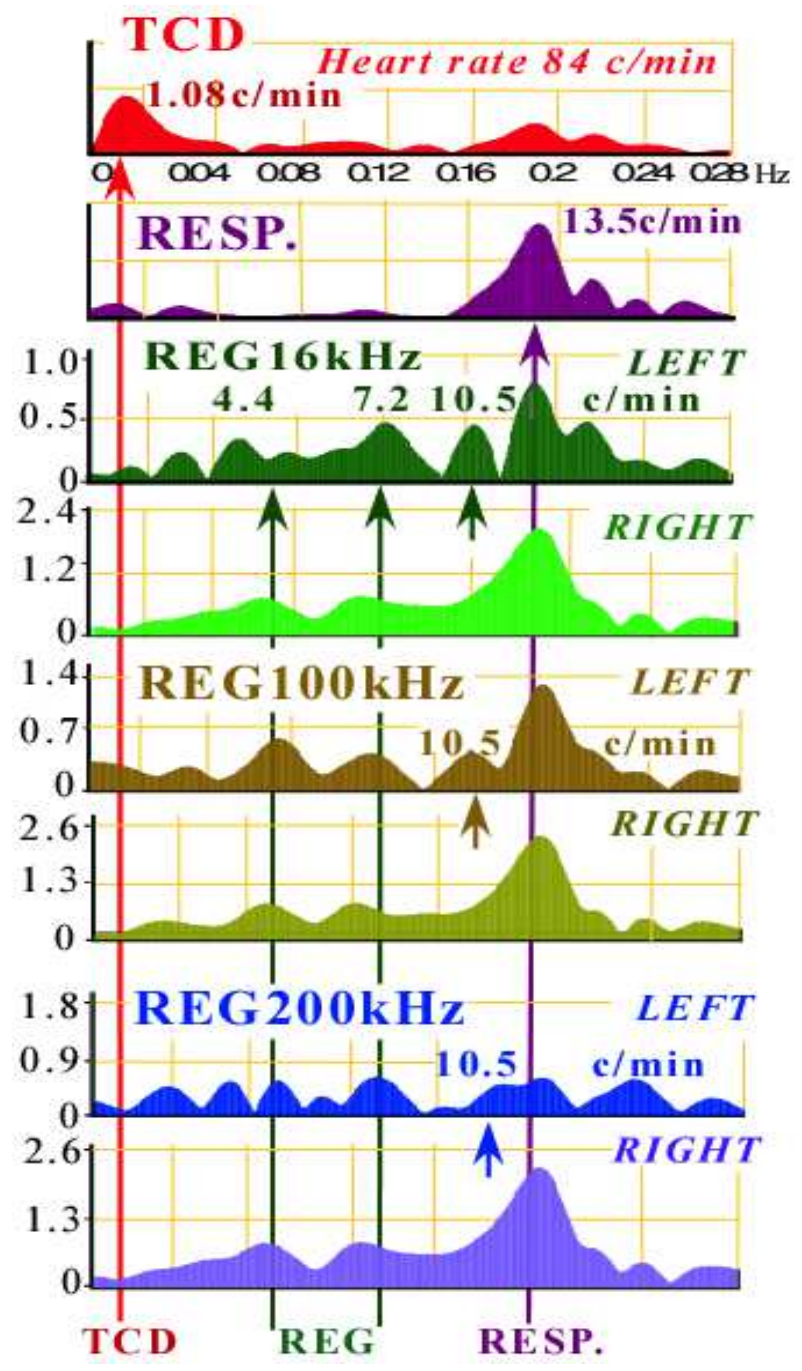

Figure 4. Spectral diagrams of slow fluctuations of REG, recorded on three different frequencies together with Transcranial Dopplerography and 
Respiratory Chest Movements.

Vertical line arrows show dominant peaks: red - TCD, green - REG, violet - respiration. Small arrows show additional peaks of REG, which there is on only one hemisphere. Blue vertical lines separate spectral diagram to sectors, where changes of some particular processes have taken place: for Sector I -TCD, Sector II - REG, Sector III Respiratory Chest Movements.

Spectral diagrams on Fig. 4 are presented by the TCD, from both hemispheres of the multi-REG and from the chest respiratory movements. Amplitude values of all spectrum fluctuations have been normalized to the maximal spectral amplitude of the cardiac cycle which is assigned the value of 1.0. Because all spectral diagrams have been normalized, every diagram is shown with its own scale on the left side. Long arrows show peaks which correspond to TCD peak (left), two REG peaks (middle) and respiratory chest movements (right). Short arrows indicate spectral lines, which are unique for only one (right or left) hemisphere. The first group of peaks with the period of $2-4$ cycles $/ \mathrm{min}$ is unique to the TCD recordings. This indicates that peaks of similar frequency on the multi-REG recordings have their origin from slow fluctuations of central arterial pressure (Traube-Hering or Mayer waves [35]. The next two or three peaks are rarely observed features unique to the multi-REG data, suggesting they originate from intracranial liquid fluctuations.

Significant differences can be observed by investigations in different age groups (Fig.5):
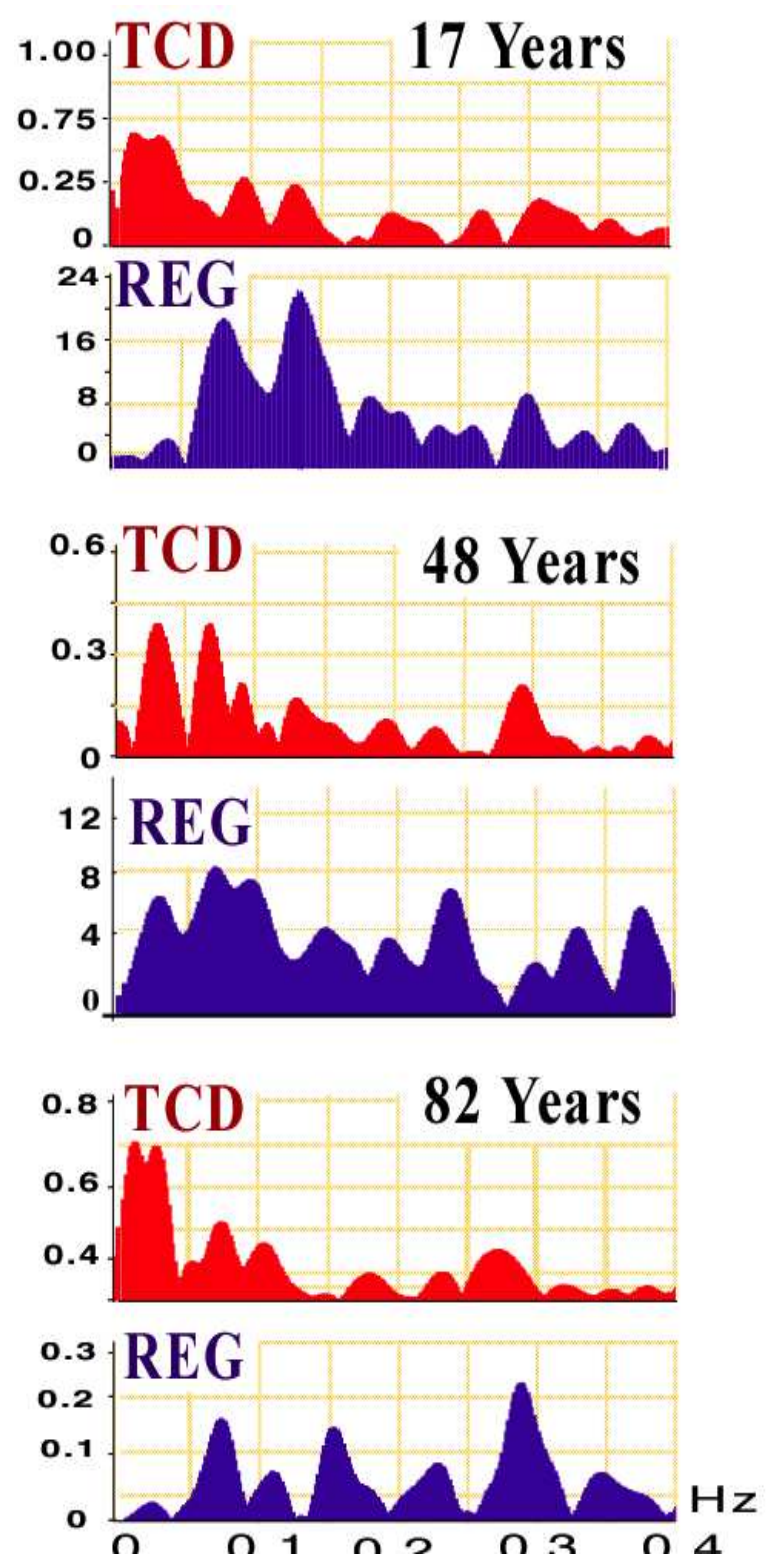

Figure 5. Spectral diagrams of TCD and REG $(100 \mathrm{kHz})$ for different age groups.

Comparative amplitudes of spectral component decrease with age and some transformation of spectral diagram components also observed, namely, maximal spectral peaks changed in direction to higher frequencies with aging, while averaged amplitudes decreased. These changes could be connected with age change of CSF - mobility and skull biomechanics. They also correspond to decrease of cognitive function of the aging brain, revealed by psychophysiological method "Prognosis", demonstrated in the previous investigations on gerontological aspects [18].

Spectral diagrams of slow fluctuations change under different physiological conditions related with control processes in the cerebro-vascular system. Changes in the spectral diagram at rest, after 5 and 10 minutes of head-down tilting in a healthy person show that at 5 minutes of this functional test some differences in Multi-REG 
$(16 \mathrm{kHz}$ and $200 \mathrm{kHz})$ increased, but at $15 \mathrm{~min}$ of the test they returned to pre-load state. Changes of TCD spectral diagrams also were observed but they not corresponded with MultiREG changes (Fig.6).

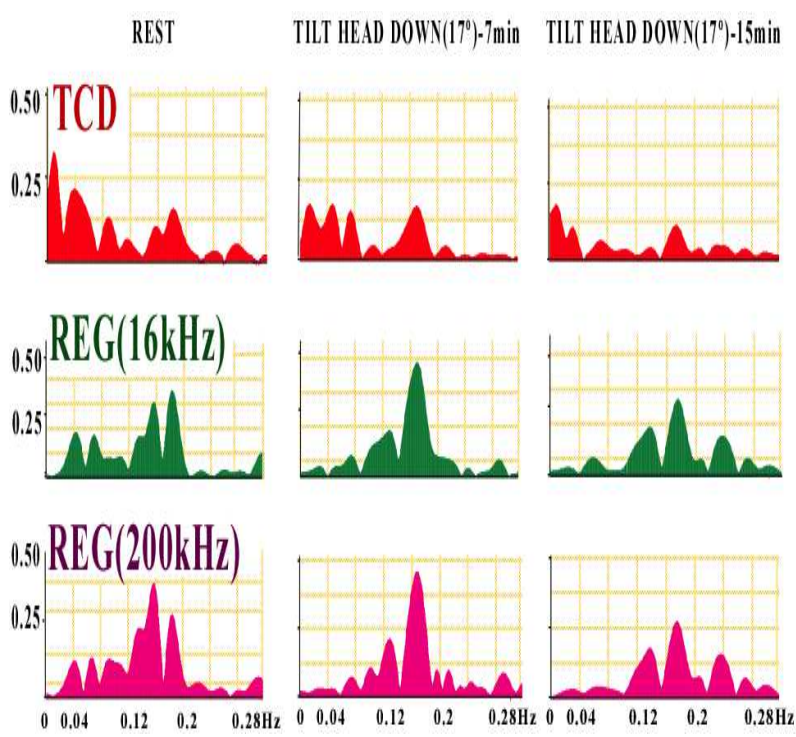

Figure 6. Changes of spectral diagram ofTCD and multiREG just before (rest) and during head-down tilt test in healthy person (24 year) - on 7th and 15th min of the test.

These data indicate that increase of blood pressure in the Upper Caval vein evokes changes in the spectrum diagram which reflects liquid volume fluctuations inside the cranium. It is notable that the effect develops during rather long time -about $20 \mathrm{~min}$. This means, that changes in intracranial venous system, as reflected by MultiREG spectral diagram, develop significantly slower to compare with responses of cerebral arteries during increase of central venous pressure. Long term changes during inhalation of hypoxic (7-9\% O2 in air) and hypercapnic (7-8\% of $\mathrm{CO} 2$ in air) gases also are reflected on low frequency spectral diagrams. However, such observations in humans may have negative influences on health. Therefore it is reasonable to provide such kinds of investigations in animal experiments.

Multi-REG investigations on animals were carried out using bi-occipital plate electrodes, placed on head of intact, awake and gently restrained rabbit. It was shown that patterns of low frequency spectral diagrams were definitely different depending on inhaled gas mixture (Fig.7):
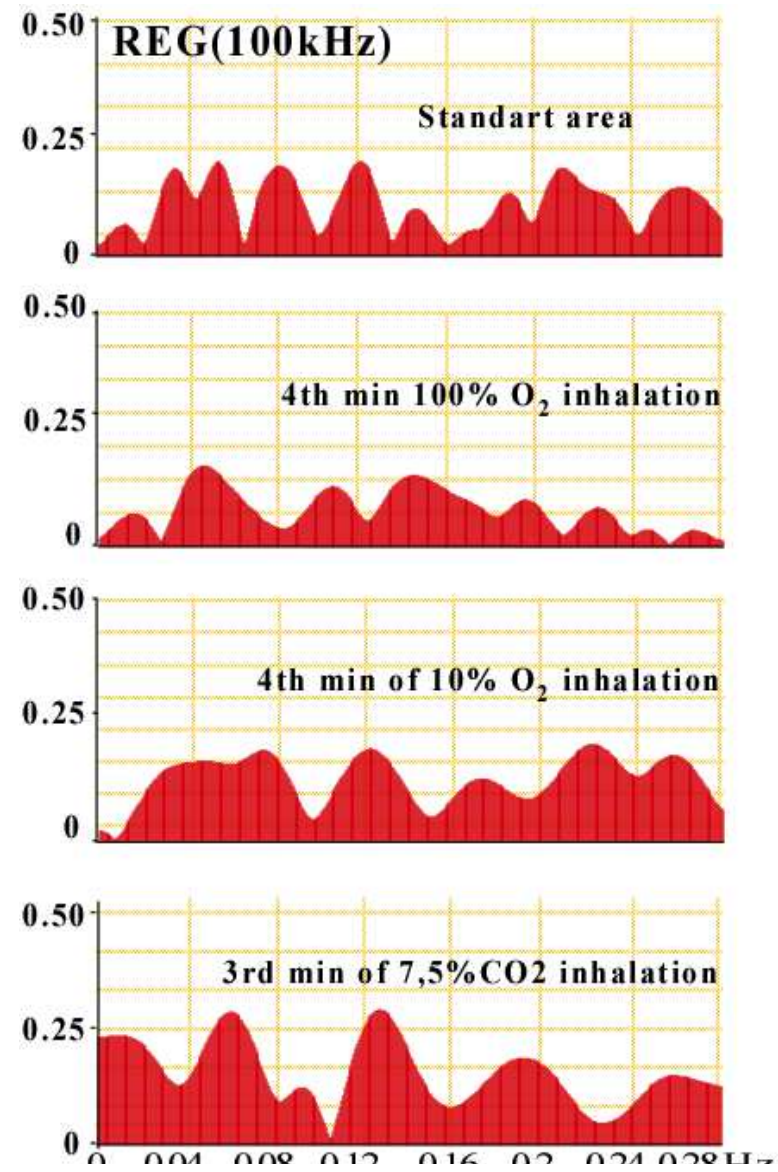

Figure 7. Spectral diagrams of intact awake animal during respiration with different gas mixtures. Upper spectral diagram represents results of normal air respiration and represents as normal conditions.

In these experiments only one frequency for REG recordings $(100 \mathrm{kHz})$ has been used, because in rabbits the spectrum diagram pattern on this frequency was found to be the most similar if to compare with human data. It id determined by the difference of electrical current distribution in head depended on skull bone thickness in human and rabbit. The investigations showed that after 3-4 minutes of gas mixture inhalation some definite changes in the spectrum diagrams of $100 \mathrm{sec}$. REG fragment could be observed. After 4 minutes of pure oxygen inhalation their line values, represented in the spectral diagram basically decreased between $20-30 \%$, particularly on frequencies of $0.2-0.3 \mathrm{~Hz}$. Inhalation of hypoxic gas is accompanied by some increase of line values (30-35\%), displayed in the spectral diagram by a decrease in the number of peaks in the analyzed spectrum interval. During inhalation of hypercapnic gas the value of spectral lines increased from $20-45 \%$, especially in frequencies $0-0.2 \mathrm{~Hz}$. The data showed that inhalation of these gas mixtures evoked definite and gas-specific changes in the low frequency spectrum. The reason for these changes in the low frequency spectrum diagram may be the specific influences of the gases on brain tissue metabolism as well as on the control mechanism for brain circulatory supply. Physiological data support both of these concepts. 
Although above presented data, concerning spectral analysis of slow fluctuations, which reflect the complicated functional physiological mechanism, responsible for circulatory-metabolic brain support, this is one of the first summarizations of experimental results of spectral analysis of slow intracranial fluctuations of non-electrical origin, and they clearly demonstrate of wide perspectives of such approach.

Slow fluctuations in $0.1-0.3 \mathrm{~Hz}$ ranges are different for every single frequency of multi-REG recordings. This means that every frequency reflects some special features of the complicated process of intracranial slow fluctuations and therefore should have its own specific informational meaning. It is important, that they are characterized by similar pattern for both humans and animals. This indicates that these fluctuations are based on processes inherent to mammalians brain.

Differences in the distribution of peaks between left and right hemispheres and some peaks appear in only a single hemisphere (mentioned on Fig.4 by short arrow). The possibility should not be excluded that hemispheric asymmetry of slow fluctuations of intracranial origin are intimately connected with hemispheric CCe and CSFm indices described earlier [30]. Therefore, there are some subtle processes which could be the basis of hemispheric asymmetry of slow fluctuations recorded by multiREG under normal physiological conditions, the nature of which is yet unclear. However, it is possible to conclude that the informational meaning of these fluctuations, which attracted the attention of specialists in some branches of medicine [27] are definitely connected with the control processes of the cerebrovascular system. Peaks at the right side of the spectral diagram of the multi-REG recordings correspond to respiratory movements of the chest and therefore reflect respiratory driven cranio-spinal liquid movements. Thus, it is possible to conclude that every fluctuation presenting in the spectral diagrams obtained from multi-REG recordings at each frequency $(16,100$ and $200 \mathrm{kHz})$ has a special, but still unclear, informational meaning. This conclusion is based on observations that under some conditions for example, after $30 \mathrm{~min}$ anti-orthostatic position, the dynamics of the spectral diagrams at different frequencies of the multi-REG change significantly.

\subsection{Peculiarities of Equivalent Circuits Components after Brain Surgery}

In contrast to healthy persons, the calculated R1, R2 and $\mathrm{C}$ components of the equivalent circuit in neurosurgical patients show changes over wide ranges depending on the kind of pathology, the surgical interventions, as well as on what postoperative day the investigation was conducted. Eight patients were investigated before and after surgery, but two typical cases each representing a different kind of pathology, were selected for the following analysis. One of them $(\mathrm{K}, 14$ years) suffered from an osteogenic tumor originated from inner plate of temporal bone which compressed the brain inward and down, and the second case ( $\mathrm{T}, 11$ years) presented with a tumor of the middle bra in which limited the CSF outflow from the brain ventricles; the tumors were removed. The results of the investigations of these neurosurgical patients show that the values of R1, R2 and $\mathrm{C}$ significantly change after surgery in comparison to presurgery measurements. These changes were most pronounced on the third and fourth day after surgery (Fig.8).

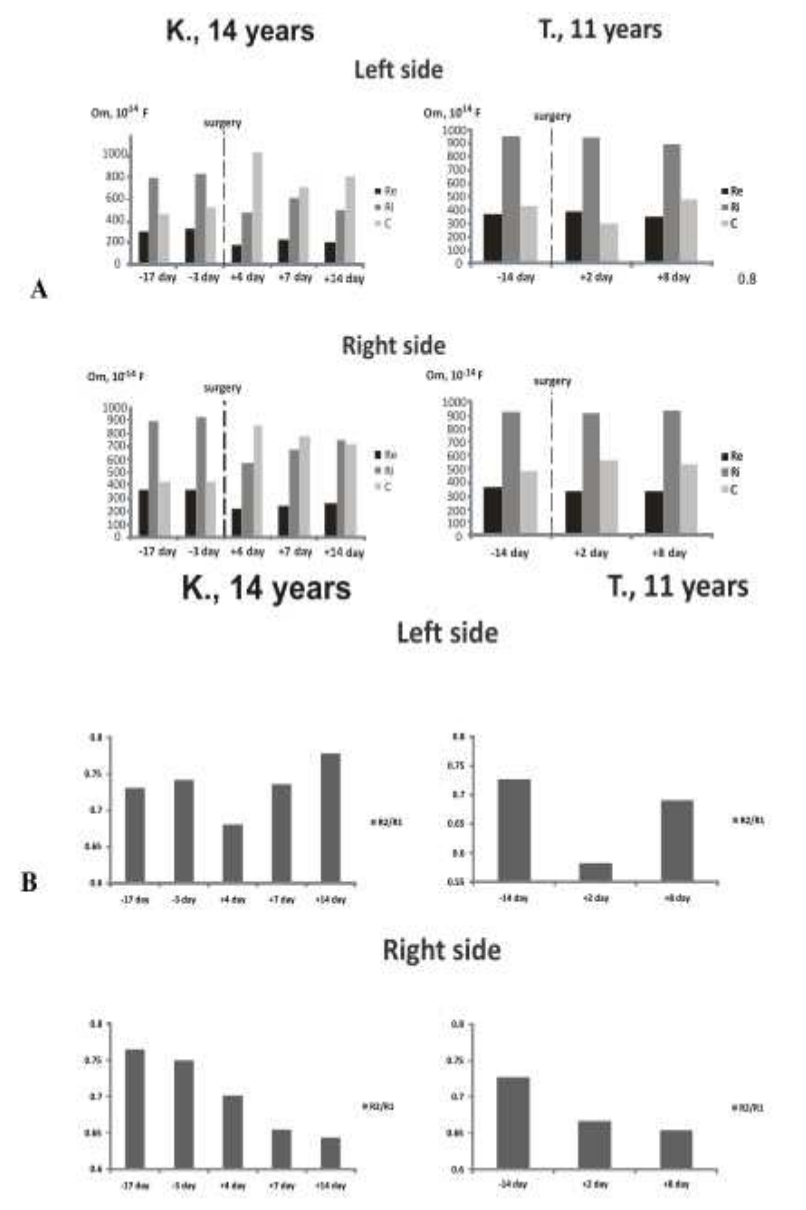

Figure 8. A-Changes of circuit I component s before and after surgery. BRatio of intracranial to extracranial resistance before and after neurosurgery

In the ensuing days the difference between pre- and post surgery R1, R2 and C values diminished as these patients gradually normalized. Similar dynamics of R1, R2, and C calculated by both circuits were observed in all the investigated patients. It is important to mention that changes of the measured indices in the operated and intact hemispheres did not appear simultaneously. These changes could be observed first in the operated hemisphere and, as these changes began to decrease, they increased in the intact hemisphere. This difference is most clearly demonstrated when comparing values of intracranial components of the equivalent circuits as a ratio in each hemisphere (Fig. 7b). So, it is possible to conclude that the indices which are comparatively stable for healthy persons change. 


\section{Discussion}

The data represented above definitely shows that multi-frequency REG has significant advantages over the usual single frequency REG. The most important advantage is that the multi-frequency REG gives the possibility to calculate resistance and capacitance elements, particular to extra-cranial and intracranial media by using calculations with two equivalent electrical circuits between electrodes. The data shows that extra-cranial resistance is less than

previously suggested and this erroneous belief, which was one reason for the limited utilization of REG during the last three decades of the Twentieth Century, is now dispelled. However, the multi-REG as was shown in the investigations of different aging groups is capable of much more subtle evaluation of the relations of liquid media inside the cranium. This is particularly evident in the results presented of investigations of different aging groups combined with results received of CSF dynamics.

Significantly the data received from the young and elderly groups have more similarities than either of them has with the middle age group. One explanation given [18] earlier for this phenomena is that for the middle age group the relations between CSF movements and cranial compliance are diminished due to decrease of articular skull bone mobility and thus a limitation of intracranial volume reserves occurs, but with age when some reduction of brain mass has taken place the reserve capacity is restored. Some increase of capacitance in the middle aging group indicates that the comparative volume of CSF and its compensative volume capabilities are minimized at this age. This is but one instance demonstrating that the sensitivity of multi-REG is significantly greater than the single frequency REG. Recently published data also suggests the multi-REG is more sensitive based on the so-called "calculated perturbative index" derived from head bioimpedance measurements [29]. By simultaneously recording the mentioned three frequencies it is possible to account for the specific current distribution of each frequency. This allows the specific contributions of the intracranial media and the extracranial tissues to be revealed. The capacitance value of the intracranial media is comparatively high and in this multi-REG model the significant role of changes in the brain tissue component can be evaluated. This is most important because it allows for examination of changes in the level of brain tissue hydration. In other words the distribution of extracellular and intracellular water can be determined.

It is known for number of decades, that practically all indices reflecting the functioning of cerebrovascular and CSF systems are not stabile at rest conditions and are characterized by slow fluctuations, initiated, besides heart and respiratory activities, also by some intracranial processes with frequency $5-15$ cycles per min $[11,27,36]$. These comparatively slow fluctuation should have significant informational meaning, because they are initiated, first of all, by processes, connected with brain tissue functioning. However, up to the recent time, it was difficult to describe these fluctuations in details because of changeable of their amplitudes and frequencies. Section 3.3 of this paper is one of the first attempts to describe quantitative statistical indices of intracranial slow fluctuations using the method of spectral analysis. REG method presents the important privilege because it permits to record these fluctuations which can be quantitatively expressed by amplitudes related to heart pulsations and by frequencies represented on spectral diagram in ranges $0-$ $0.3 \mathrm{~Hz}$, where the most low frequencies of the fluctuations are localized. Of course, it is impossible now to describe all features of these fluctuations, but some of them become clear. Firstly, slow fluctuations, recorded by REG, reflect mainly intracranial volume fluctuations, which connected with cerebrovascular control processes and brain metabolism. Secondly, they are connected, but different from control processes in cardiovascular system. Thirdly, correlation of amplitude and frequency distribution of spectrum lines in different aging groups indicate, that slow fluctuations of intracranial origin indirectly reflect brain cognitive activity. Fourthly, they are similar for human and for mammalian representative - rabbit, suggesting that such slow fluctuations reflect metabolic processes common for mammalian brains. All above demonstrate, that the use of REG method is of perspective for brain investigations both in fundamental and applied aspects.

Numerous animal studies investigating the electrical properties of brain tissue and their electrical parameters established that a close connection exists between brain tissue hydration and its electrical parameters. These studies also established that a unique ratio exists between the resistance of brain tissue and its capacitance. [23, 24]. Similar conclusions were reached for the human brain during investigations of brain cellular metabolism. It was shown that the common factor in brain injuries of various causes is actually cellular injury. The injuries affect the cellular metabolism, especially the aerobic oxidative respiration of the cell [37]. This reduces the cell's capacity to produce energy forces to the cell membrane which leads to the accumulation of active osmotic products in the intracellular space. This results in water accumulation and intracellular swelling or cytotoxic edema [38] as in the early stages of hypoxic ischemia. As a consequence of the edema the electrical parameters of the brain tissue change [39, 40, 41]. This group of investigations establishes that dramatic changes in intracranial electrical parameters accompany most brain injuries. Thus it could be expected that multi-REG monitoring of brain injured patients would likely show changes of intracranial electrical parameters.

It is clearly possible to see such changes in the data received during our investigations of neurosurgical patients (fig.8). In patient K. an osteogenic tumor originated from the inner plate of left temporal bone. Patient $\mathrm{T}$. was diagnosed with intracerebral tumor which compressed and displaced the brain resulted in hydrocephalic syndrome. These examples provide two interesting points: first, the maximal changes of intracranial resistance develop on the 
second and third day after surgery, which corresponds to the general clinical observation of the patients; secondly the maximal changes of electrical indices occurred not simultaneously in both hemispheres. The changes of electrical data were observed first on the operated hemisphere and then later on the intact hemisphere.

Thus, observations on healthy persons of different ages as well as on neurosurgical patients show that significantly valuable data can be obtained noninvasively by means of the multi-REG that relates to the properties of the brain tissue, particularly its hydration state.

\section{Conclusions}

The method of rheoencephalography is not new. It has progressed with time and technical developments. Its long history is characterized by periods of euphoric enthusiasm and dramatic disappointments. During its inception, enthusiasm for the possibility of noninvasive study of brain blood circulation was high and numerous papers using REG were published. The enthusiasm was dampened over time as the investigations of numerous of skeptics demonstrated this method could not be used for cerebral blood flow measurements. The redemption for REG was its marriage to TCD, which then opened new possibilities for the study of CSF dynamics. Another significant step forward came during numerous animal investigations which initiated the idea to use multifrequency measurements. However, between the idea and its realization were years of delay awaiting innovations in microelectronics and computer technology. Finally the possibility arrived to noninvasively monitor the condition of brain tissue, primarily the level of its extracellular and intracellular hydration. Simultaneously recording the multi-REG with TCD provided the possibility to monitor some indices of CSF dynamics along with brain tissue hydration. The REG/TCD combination significantly enlarged the horizons for experiment and clinical application of this method. The comparatively high sensitivity of the multi-REG and the wide variety of indices reflected in the recordings, including slow fluctuations, suggests that such a methodology can detect subtle changes of brain circulatory metabolic supply, which may correspond to even very slight changes of brain cognitive function. Therefore it looks reasonable in perspective to combine multi-REG investigations with some method, which could dynamically reflect changes of brain cognitive function by some psycho-physiological used.

Possibilities of multi-REG have not been fully examined. The most interesting data was received by spectral analysis of the pulse fluctuations from the REG, recorded at different frequencies as shown in Fig. 4 This figure presents a number of unexpected new elements. The spectrum in ranges $0-0.3$ $\mathrm{Hz}$ reflects the control processes in the cerebrovascular system, as was previously suggested, but here the recordings present much more information than was thought possible. Indeed, the spectral diagrams of different frequencies are individually unique, which suggests that every frequency reflects its own unique influence to the complicated picture of the real control system supporting the circulatory-metabolic brain system. It is most interesting that it is possible to observe left-right asymmetry in slow fluctuations particularly in some groups of spectral lines which occur in only one hemisphere (shown in Fig.4 by short arrows). These anomalies are at the present time terra incognita in the realm of slow fluctuations.

It is important, that spectrum diagram of slow fluctuation of intracranial origin is similar for human and representative of mammalians. This, firstly, is one more evident that REG method could reflect namely intracranial fluctuating processes, and, secondly, opens the way to provide complex investigations of some neurological problems, with additional experimental animal models for some pathological conditions. This is perspective also for some pharmacological studies, for pre-clinical investigation of drug action in animal pathophysiological models. Generally, it is possible to conclude that in our time the dynamic modification of REG method, based on simultaneous measurements at three frequencies, will ascend to new heights and issue in a "Renaissance" period for noninvasive monitoring of the cerebral vascular system, including CSF dynamics and the properties of the brain tissue. It may even surpass computer tomography by virtue that the information is more exact and clinically more useful as well as transportable, easy to use and vastly less expensive than comparable methods.

Supported by Grant RFFI No.13-04-00612

\section{References}

[1] Kety SS, Schmidt CF, "Cerebral blood f low in man", Amer. J. Physiol., v.27, pp.53-66, 1945.

[2] Nyboer J, Electrical impedance plethysmography, Springfield: Charles C. Thomas, 1959.

[3] Schwan HP, "Determination of biological impedances", In: Physical techniques in biological research. N.Y.- L. V.6. pp. 323-407, 1963.

[4] Kedrov FA, Naumenko AI, Physiology of intracranial circulation and its clinical aspects, Leningrad, 1954. (In Russian)

[5] Pratesi F, Nuti A, Sciagra A, "Reografia cranica", Minerva Medica., V.48, No.7, pp.3223-3229, 1957.

[6] Jenkner F, Rheoencephalography, Springfield, Charles T. Thomas, 1962.

[7] Seipel JH, Liemnowicz SA, O'Doherty PS, "Cranial impedance plethysmography - rheoencephalography as a method of detect ion of cerebrovascular disease" In: Cerebral Ischemia. Springfield. pp.162-180, 1964.

[8] Moskalenko YuE, Naumenko AI, "About oscillatory movements of CSF in craniospinal cavity", Physiol. J. USSR, V.43, No.10, pp.928-933, 1957.

[9] Moskalenko YuE, Naumenko AI, "Investigation of CSF 
translocations in normal animals", Physiol. J. USSR, V.45, No.5, pp.562-568, 1959.

[10] Seoane F, Lindecrantz K, Olsson T, Kjellmer I, Flisberg A, Bågenholm R, "Spectroscopy study of the dynamics of the transencephalic electrical impedance in the perinatal brain during hypoxia", Physiol. Meas, V. 26, pp.849-863, 2005.

[11] Moskalenko YuE, Dynamics of the brain blood volume under normal conditions and gravitational stresses, Nauka Press, Leningrad, 1967. (English translation: NASA-TT F-492)

[12] Yarullin HH, Clinical rheoencephalography, Moscow, 1967.(In Russian).

[13] Moskalenko YuE, Weinstein GB, "Rheoencephalography: Biophysical basis, informative value, the limits of application", Human Physiology, v.9, No.5, pp.707-722, 1983.

[14] Moskalenko YuE, Weinstein GB, Semernia VN, "Investigation of human cerebral circulation in spaceflight conditions", Aviat . Space Environ. Med. V.46, No.8, pp.1023-1027, 1975.

[15] Moskalenko YuE, Weinstein GB, Semernia VN, "Functional stability of cerebrovascular system to space flight conditions", In: Biological research on the "Salut" orbital stations. Nauka-Press: Moscow, pp.144-151, 1984.

[16] Bodo M, "Studies in Rheoencephalography (REG)", J. Electr. Bioimp., v.1. pp.18-40, 2010.

[17] Aaslid R, Transcranial Doppler sonography. Springer-Verlag. N.Y., 1986

[18] Moskalenko YuE, Ryabchikova NA, Weinstein GB, Halvorson P, Vardy TC, "Changes of circulatory-metabolic indices and skull biomechanics with brain activity during aging", J. Integrative Neuroscience, v.10, No 2. pp.131-160, 2011.

[19] Moskalenko Yu., Weinstein G., Masalov I., Ryabchikova N., Halvorson P., Semernia V., Andreeva Yu., Panov A., "Multifrequency REG: Fundamental background, informational meaning and ways of data analyzing" Am .J. Biomed. Eng. V.2, No.4, pp. 163 - 174, 2012.

[20] Masalov I. S., Andreeva Yu. V., Erkebaeva S. K., Weinstein G.B. "A method of automatized determination of parameters of intracranial liquorodynamics by data of rheoencephalogram and transcranial dopplerogram", J. of Evolutionary Biochemistry and Physiology. V. 49, No 1, pp. $85-87,2013$

[21] Moskalenko YuE, Weinstein GB, Halvorson P, Kravchenko TI, Feilding A, Ryabchikova NA, Semernia VN, Panov AA, "Age peculiarities of relationships between brain blood flow, liquor dynamics and biomechanical properties of human cranium”, Russian Physiol. J., v.93, No 7. pp.788-798, 2007.

[22] Zaks M, Moskalenko Yu, "Electrical characteristics of the mammary gland of women in the first days of lactation", Physiol. J. USSR, v.48, No7, pp.850-855, 1962 (translated to English by Federation Proc. 1963, pp.969-973).

[23] Moskalenko YuE, Vorobiev MV, Weinstein GB, "Electrical impedance techniques for monitoring water balance in the brain". Proc. of the Annual Conference of IEEE Engineering in Medicine and Biological Society, v.13, No.4,pp.1549
$-1550,1991$

[24] Moskalenko YuE, Beketov AI, Weinstein GB, "The involvement of cerebrovascular reactivityin pathogenesis of space mot ion sickness", Acta Astronautica, v.23, pp.97-103, 1991

[25] Seoane F, Lindecrantz K,"Extract ion of structural information from impedance spectrum data. A step towards the identification of cellular oedema", Proc. of the 18the EURASIP Biosignal Conference. Analysis of Biomedical Signals and Images, Brno, pp.90-93, 2006.

[26] Schwan H, "Alternative current spectroscopy of biological substances", Proc. of the Institute of Radio Engineers (IRE), v 47, No.11, pp.1841-1855, 1959.

[27] Moskalenko YuE, Kravchenko TI, "Wave phenomena in movements of intracranial liquid media and the primary respiratory mechanism", Amer Acad. Osteopat. J., v.14, pp.29-40, 2004.

[28] Wang J, Yang H, Wang P, Dong W, "Noninvasive monitoring of hypertensive intracerebral hemorrhage and perihematomal tissue edema”, Natl. Med. J., China, v.83 pp.471-474, 2003

[29] Liu LX, Dong WW, Wang J, Wu Q, He W, Jia YJ, “The role of noninvasive monitoring of cerebral electrical impedance in stroke", Acta Neurochir., Suppl. 95, pp.137-140, 2005.

[30] Moskalenko YuE, Weinstein GB, Ryabchikova NA, Halvorson P, Feilding A, Kravchenko TI, Vardi T, Samus NL, Semernia VN, Panov AA, "Interhemispheric asymmetry of the CSF dynamics and biomechanical properties of the skull", Russian J. of Physiology, v.96, No.10, pp.1005-1013, 2010.

[31] Courchesne E, Chisum HJ, Townsend J, Cowless A, Covington J, Egaas B, Harwood M, Hinds S, PressGA, "Normal brain development and aging: Quantitative analysis of in vivo MR imaging in healthy volunteers", Radiology, v.216, No.3, pp.672-682, 2000.

[32] Zhang L, Thomas KM, Davidson MC, Casey BJ, Heier LA, Ulug AM, "MR quantitation of volume and diffusion changes inthe developing brain", Amer. J. of Neuroradiology, v.26, No.1, pp.45-49, 2005

[33] Hill R, Jansen J, Fling J, "Electrical impedance plethysmography: a critical analysis", J. App 1. Physiol., v.22, No.1, pp.161-168, 1967.

[34] Eremeev VS, Semeniutin VB, Tashaev SS, Teplov SI, "The informativity of rheoencephalogram dynamics", Physiol. J. USSR, v.64, No.8, pp.1145-1152, 1978.

[35] Koepchen HP, "History of studies and concepts ofblood pressure waves", In: Mechanisms of blood flow pressure waves", (Eds. Miyakawa K et al). Berlin:Springer-Verlag, pp. 3-23, 1984.

[36] Moskalenko Yu, Cooper R, Crow H, Walter WG, "Variation in blood volume and oxygen availability in the human brain" Nature, v.172, No.4928, pp.159-161, 1964.

[37] Cotran RS, "Endothelial cells" In: Kelly's Textbook of Rheumatology", Saunders Company, Philadelphia, pp.381-415, 1989 .

[38] Klatzo I, "Evolution of brain edema", Acta Neurochir., Suppl. (Wien), v. 60, pp.3-6, 1994. 
[39] Ochs S, Van Harreveld A, "Cerebral impedance changes after circulatory arrest", Amer. J. Physiol, v.187, No.1, pp.180-192, 1956.

[40] Holder DS, "Electrical impedance tomography with cortical or scalp electrodes during global cerebral ischemia in anaesthetized rat", Clin. Phys. Physiol. Meas., v.13, No.1, pp.87-98, 1992.
[41] Lingwood BE, Dunster KR, Golditz PB, Ward LC, "Noninvasive measurement of cerebral bioimpedance for detect ion of cerebral edema in the neonatal piglets" Brain Res., v.945, No.1, pp.97-105, 2002. 